\title{
EFFECTS OF MUSIC THERAPY UNDER GENERAL ANESTHESIA IN PATIENTS UNDERGOING ABDOMINAL SURGERY
}

\author{
Nakhli M.S., Kahloul M., Chakroun A., Harrathi M.A., Mhamdi S. \\ Sahloul Hospital, Dept of Anaesthesiology \& Intensive Care, Sousse, Tunisia
}

\section{Introduction:}

Music therapy, an innovative approach that has proven effectiveness in many medical conditions, seems beneficial also in managing surgical patients. The aim of this study is to evaluate its effects, under general anesthesia, on perioperative patient satisfaction, stress, pain and awareness.

\section{Methods:}

This is a prospective, randomized, double-blind study conducted in the operating theatre of visceral surgery at Sahloul teaching hospital over a period of 4 months. Patients aged more than 18, undergoing a scheduled surgery under general anesthesia were included. Patients undergoing urgent surgery or presenting hearing or cognitive disorders were excluded. Before induction, patients wore headphones linked to a MP3 player. They were randomly allocated into 2 groups: Group M (with music just after anesthesia induction until the surgery end) and group C (without music). Hemodynamic parameters, quality of arousal, pain experienced, patient's satisfaction and awareness incidence during anesthesia were recorded.

\section{Results:}

One hundred and fourty patients were included and allocated into 2 groups which were comparable in demographic characteristics, surgical intervention type and anesthesia duration.

The comparison of these two groups, regarding the hemodynamic profile, found more stability in group $\mathrm{M}$ for systolic arterial blood pressure. (Figure 1)

A calm recovery was more often noted in group $M(77.1 \%$ versus $44.2 \%, p<10^{-3}$ ). The average Visual Analog Scale (VAS) was lower in the intervention group (33.8 \pm 13.63 versus $\left.45.1 \pm 16.2 ; p<10^{-3}\right)$. The satisfaction rate was significantly higher in experimental group $(81.4 \%$ versus $51.4 \%$; $p<10^{-3}$ ).

The incidence of intraoperative awareness was higher in group C (8 cases versus 3 cases) without statistically significant difference. (Table 1)

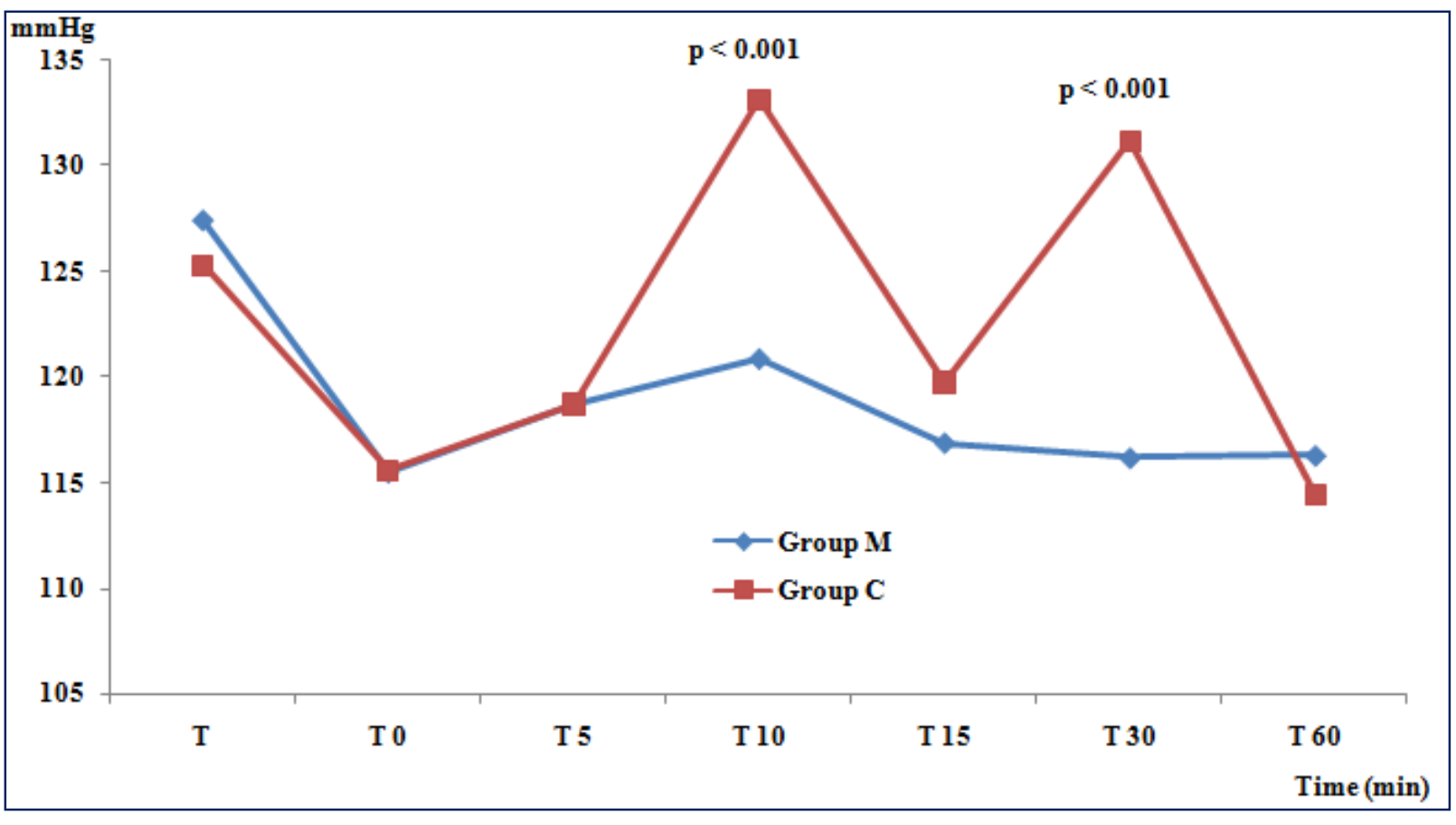

Figure 1 : changes, in systolic blood pressure, relates to music therapy

\section{Discussion:}

Music is a very old therapeutic mean whose effectiveness was essentially proven in the treatment of physical and mental stress related to certain neuropsychiatric disorders.

We found a significant improvement in patient satisfaction. Dubois JM assessed this effect for patients scheduled for bronchoscopy. The satisfaction was significantly greater in the intervention group (1). The same results were found by a recent meta-analysis including 712 patients undergoing colonoscopy under general anesthesia. Music therapy improves satisfaction directly by its relaxing effect, and indirectly through its effects on other dissatisfaction factors such as perioperative pain and stress and postoperative nausea and vomiting. This effect is seen essentially when the music used is chosen by the patient. Another beneficial effect of music therapy was intraoperative hemodynamic stability. Binns-Turner PG and al demonstrated this effect for MAP (2) while Jaber and al demonstrated it for HR and SAP (3).

\section{References:}

Dubois JM, Bartter T, Pratter MR. Music improves patient comfort level during outpatient bronchoscopy. Chest. $1995 \mathrm{Jul}$

Binns-Turner PG, Wilson LL, Pryor ER and al. Perioperative music and its effects on anxiety, hemodynamics, and pain in women undergoing mastectomy. AANA J. 2011.

. Jaber $\mathrm{S}$, Bahloul $\mathrm{H}$, Guétin and al. Effects of music therapy in intensive care unit without sedation in weaning patients versus non-ventilated patients. AFAR 2007

. Jayaraman L, Sharma $\mathrm{S}$, Sethi $\mathrm{N}$ and al. Does intraoperative music therapy or positive therapeutic suggestions during general anesthesia affect the postoperative outcome ? - a double blind randomised controlled trial. Indian J. Anaesth. 2006.
The mechanism of action would be a modulation of neuro-hormonal response.

In our study, the recovery quality was better in the intervention group showing a significant reduction in the level of stress and anxiety. According to Palmer JB and al, perioperative anxiety is due to a conflict between a real event and a scheduled event that will activate the sympathetic nervous system to bring up the signs of anxiety. Intraoperative music therapy, when it is chosen by the patient, will act by modulation of this conflict, which will give a relaxation by activating the parasympathetic nervous system .

Table 1 : Effects of music therapy in recovery quality, VAS, patient satisfaction and intraoperative awareness

\begin{tabular}{lccc}
\hline \multicolumn{1}{c}{ Studied parameters } & Group M & Group C & p \\
\hline Recovery quality: & & & \\
\hline - calm & $77.1 \%$ & $44.2 \%$ & $<10^{-3}$ \\
- agitated & $22.8 \%$ & $55.7 \%$ & \\
VAS during recovery & & & \\
- <3 & $51.4 \%$ & $30 \%$ & \\
- 3 to 6 & $42.8 \%$ & $51.4 \%$ & 0.01 \\
- >6 & $5.7 \%$ & $18.5 \%$ & \\
Patient satisfaction & $81.4 \%$ & $51.4 \%$ & $<10^{-3}$ \\
Intraoperative awareness & $4.2 \%$ & $11.4 \%$ & 0.10 \\
\hline
\end{tabular}

Intraoperative music therapy also significantly reduced the pain intensity. Jayaraman $L$ and al found similar results in 111 patients undergoing laparoscopic surgery (4). The mechanism of action is multifactorial involving an attenuation of the conduction in the afferent fibers, mnemonic encoding, stimulation of endorphin production and action on psychomotility.

The last point we tried to search is the relationship between music therapy and intraoperative awareness. This complication is common and serious because of its psychological consequences. Its incidence was less frequent in the intervention group but the difference was not significant.

\section{Conclusion:}

Music therapy is a non-pharmacological, inexpensive and noninvasive technique that can significantly enhance patient satisfaction and decrease patients' embarrassing experiences related to perioperative stress, pain and awareness. 